\title{
Efectos de dos anticoagulantes sobre el recuento celular y parámetros de activación plaquetaria de plasma rico en plaquetas de equinos
}

\author{
Effect of two anticoagulants on the cell count and platelet activation parameters \\ from equine platelet-rich plasma \\ CE Giraldo*, C López, JU Carmona \\ Grupo de Investigación Terapia Regenerativa, Departamento de Salud Animal, Universidad de Caldas, Manizales, Colombia.
}

\begin{abstract}
SUMMARY
The aims of the study were 1) to compare the effect of the anticoagulants, sodium citrate (CS) and acid-citrate-dextrose (ACD) solution A (ACDA) on counts of platelets (PLTs) and leukocytes and on the activation platelet-related variables, mean platelet volume (MPV) and platelet distribution width (PDW) in whole blood, platelet-rich plasma (PRP) and platelet-poor plasma (PPP) from equines; 2) To set the collection efficiencies for PLT and leukocytes in PRPs obtained with both anticoagulants and 3) to correlate PLT and leukocyte counts, MPV and PDW in every blood component. Whole blood from 18 horses was collected in both CS and ACD-A tubes, PRP and PPP were obtained by the tube method. Whole blood, PRP and PPP were evaluated by automated haemogram. PLT and leukocyte counts in whole blood, PRP and PPP were not affected by anticoagulants. PLT counts were significantly higher $(\mathrm{P}<0.001)$ in PRP compared to whole blood and PPP. Leukocyte counts were similar between whole blood and PRP, but differed significantly $(\mathrm{P}<0.001)$ from PPP. No significant differences were observed for MPV and PDW between whole blood and PRP. Significant correlations were observed between PLT and leukocyte counts $(\rho: 0.73, \mathrm{P}<0.01)$, leukocyte and lymphocyte counts $(\rho: 0.76, \mathrm{P}<0.01)$ and PLT and lymphocyte counts $(\rho: 0.60, \mathrm{P}<0.01)$. A negative moderate correlation between leukocyte counts and PDW ( $\rho:-0.65, \mathrm{P}<0.01)$ was found. The anticoagulant used for producing equine PRP had no effect on cell concentration and activation platelet-related parameters. Thus, cell parameters are no useful for discriminating which of both anticoagulants could be better for producing equine PRP.
\end{abstract}

Key words: equine, platelet-rich plasma, regenerative medicine, sodium citrate, ACD-A.

\section{RESUMEN}

Los objetivos del estudio fueron 1) comparar el efecto de los anticoagulantes citrato de sodio (CS) y ácido-citrato-dextrosa (ACD), solución A (ACDA), sobreconteos de plaquetas (PLTs), leucocitos y variables de activación plaquetaria, volumen plaquetario medio (VPM) y ancho de distribución del tamaño de las plaquetas (ADTP) en sangre total, plasma rico en plaquetas (PRP) y plasma pobre en plaquetas (PPP) de equinos; 2) establecer eficiencias de colección de PLT y leucocitos para PRP obtenidos con ambos anticoagulantes y 3) correlacionar recuentos de PLT, leucocitos, VPM y ADTP de cada componente sanguíneo. Se recolectó sangre de 18 caballos con tubos de CS y ACD-A, elaborando PRP y PPP por cada anticoagulante por el método del tubo. Sangre total, PRP y PPP fueron evaluados mediante hemograma automatizado. Los conteos de PLT y leucocitos en sangre total, PRP y PPP no fueron afectados por ambos anticoagulantes. Conteos de PLT fueron significativamente mayores $(\mathrm{P}<0,001)$ en PRP comparados con sangre total y PPP. Los conteos de leucocitos fueron similares entre sangre total y PRP, difiriendo significativamente $(\mathrm{P}<0,001)$ del PPP. No hubo diferencias significativas para VPM y ADTP entre sangre total y PRP. Se observaron correlaciones significativas entre conteos de PLT y leucocitos $(\rho: 0,73, P<0,01)$, conteos de leucocitos y linfocitos ( $\rho: 0,76, \mathrm{P}<0,01)$, conteos de PLT y linfocitos $(\rho: 0,60, \mathrm{P}<0,01)$, y correlación negativa moderada entre conteos de leucocitos y $\operatorname{ADTP}(\rho:-0,65, P<0,01)$. Los anticoagulantes utilizados en la preparación del PRP equino no influyeron sobre concentración celular ni parámetros de activación plaquetaria. Así, los parámetros celulares no son útiles para discriminar cuál de los dos anticoagulantes es mejor para producir PRP equino.

Palabras clave: caballo, plasma rico en plaquetas, medicina regenerativa, citrato de sodio, ACD-A.

\section{INTRODUCCIÓN}

La aplicación de concentrados autólogos de plaquetas (PLT) ha comenzado a ser un tratamiento común en equinos con afecciones locomotoras y heridas, entre otros (Carmona y col 2009a , Carmona y col 2009 , López y col 2010, Carmona y López 2011, Iacopetti y col 2012, Carmona y col 2013a , López y Carmona 2014). Estos

Aceptado: 19.03.2015.

* Calle 65 N $^{\circ}$ 26-10, Manizales, Colombia. E-mail: carmona@ucaldas. edu.co concentrados, según la cantidad de PLT y leucocitos contenidos, se denominan plasma rico en plaquetas puro (PRP-P) y plasma rico en plaquetas y leucocitos (PRP-L) (McCarrel y Fortier 2009, Dohan Ehrenfest y col 2012, Carmona y col 2013 ${ }^{\mathrm{b}}$ ).

El PRP leucocitos-reducidos puede ser la preparación óptima para estimular la reparación tisular sin formación de tejido cicatricial (McCarrel y col 2012). La concentración alta de leucocitos absolutos en PRP equino contribuye a la expresión de citocinas inflamatorias que pueden retardar la regeneración celular y estimular la formación de tejido cicatricial (McCarrel y col 2012). Por lo anterior, es importante buscar un anticoagulante 
apropiado para evitar la activación plaquetaria espontánea durante la preparación de PRP para utilización terapéutica o experimental (Lei y col 2009), y además relacionar la concentración de plaquetas/ $\mu \mathrm{L}$ con la concentración de leucocitos/ $\mu \mathrm{L}$ del PRP.

Las plaquetas presentes en el PRP son una fuente particular de varios factores de crecimiento (FC) almacenados en sus gránulos alfa. Estas incluyen el FC derivado de plaquetas, FC transformante beta, FC endotelial vascular, FC similar a insulina tipo I, FC epidérmico y FC de hepatocitos, entre otros (Marx 2004, Sutter y col 2004, Carmona y col 2011). Las plaquetas concentradas aparentemente proveen concentraciones suprafisiológicas de FC que aceleran la regeneración celular y la reparación de tejidos lesionados (Marx 2004, Sutter y col 2004, Carmona y col 2011).

Los anticoagulantes inhiben la coagulación y agregación de las PLT durante la preparación de un PRP con fines terapéuticos (Araki y col 2012). Ellos permiten mantener los PRP en estado líquido para su posterior aplicación en las lesiones. Sin embargo, son escasas las investigaciones acerca del efecto de los anticoagulantes respecto de la concentración de PLT y leucocitos y el efecto de los parámetros de activación plaquetaria, como el volumen plaquetario medio (VPM) y el ancho de distribución del tamaño de las plaquetas (ADTP) en PRP equino y de otras especies, incluyendo la humana (Lei y col 2009). En caballos es aún un tema de debate la selección del anticoagulante para la preparación de PRP. Según la literatura revisada, no se encontraron reportes científicos completos relativos al efecto de los anticoagulantes en PRP obtenido mediante métodos manuales, posiblemente por el alto costo que tienen los materiales, las pruebas moleculares y, también, por la falta de interés de los fabricantes de los aparatos disponibles comercialmente para la preparación de PRP en caballos (Carmona y col 2013 ${ }^{\text {b }}$ ).

Los anticoagulantes citrato de sodio (CS) y ácido citrato dextrosa solución A (ACD-A) son los más comúnmente utilizados para la preparación de PRP equino con fines terapéuticos (Schnabel y col 2007, López y col 2010, Carmona y López 2011, Castelijns y col 2011). Estos producen una acción antiagregante y evitan la activación plaquetaria. Actualmente se reconoce que el efecto de los anticoagulantes sobre la activación plaquetaria es un área de preocupación; por ejemplo, si los anticoagulantes con mecanismo de acción igual o diferente no pueden impedir o aún pueden promover la activación espontánea de las plaquetas ex vivo (Lei y col 2009).

Tradicionalmente, el PRP equino con propósitos experimentales o clínicos se prepara con sangre recolectada con citrato de sodio (CS) o ACD-A sin consideraciones manifiestas que justifiquen la elección de uno u otro anticoagulante. Los anticoagulantes se utilizan comúnmente en métodos manuales con tubos de CS (tapón azul) (Argüelles y col 2006, Maia y col 2009, Monteiro y col 2009, Álvarez y col 2011, Dunkel y col 2012, Vendruscolo y col 2012), y con tubos ACD-A (tapón amarillo) (Tablin y col 2008, Textor y Tablin 2012, Giraldo y col 2013), filtración gravitacional con ACD (Castelijns y col 2011), sistemas semiautomatizados o aparatos con CS (Sutter y col 2004, Textor y col 2011) y con ACD-A (Schnabel y col 2007), y aféresis con CS (Sutter y col 2004) y con ACD-A (Carter y col 2003). Esta última técnica permite separar los componentes de la sangre, seleccionar los necesarios para su aplicación terapéutica y devolver el resto de componentes al torrente sanguíneo.

Las PLT pierden su capacidad para agregarse cuando se les priva de cationes divalentes como $\mathrm{Ca}^{2+}$ (Zucker y Grant 1978). También, el pH y la temperatura durante la incubación influyen directamente en el grado de inhibición de agregación de las PLT (Zucker y Grant 1978). El cambio de $\mathrm{pH}$ del plasma dependiente del anticoagulante utilizado podría afectar la adhesividad de las plaquetas (Flatow y Freireich 1966). La activación de plaquetas exhibe un requerimiento bifásico de calcio: el incremento transitorio inicial en concentración de calcio intracelular ( $\left.\left[\mathrm{Ca}^{2+}\right] \mathrm{i}\right)$ proviene de depósitos intracelulares, mientras que el segundo paso de degranulación puede ser únicamente si hay $\mathrm{Ca}^{2+}$ extracelular disponible (Davies y col 1989). Por tanto, la concentración de $\mathrm{Ca}^{2+}$ total en el PRP puede afectar el grado de activación in vitro de las PLT. Los cambios en concentración de $\mathrm{Ca}^{2+}$ extracelular afectan directamente los resultados de las pruebas de la función plaquetaria (Kingston y col 2001) y posiblemente podrían afectar la concentración celular y los parámetros de activación de las PLT contenidas en el PRP.

Los objetivos del presente estudio fueron: 1) Comparar el efecto de los anticoagulantes citrato de sodio (CS) y ácido-citrato-dextrosa, solución A (ACD-A) sobre los recuentos de PLT, leucocitos y sobre el VPM y el ADTP en sangre total, PRP y PPP de equinos obtenidos mediante una técnica de doble centrifugación en tubo. 2) Correlacionar de manera general los recuentos de PLT, leucocitos y los valores de activación plaquetaria de cada componente sanguíneo. Nuestra hipótesis fue que los anticoagulantes no podrían influir sobre la composición celular o los parámetros de activación plaquetaria en el PRP equino.

\section{MATERIAL Y MÉTODOS}

El presente estudio fue aprobado por el Comité de Ética de la institución de los autores.

\section{CABALLOS}

Fueron utilizados dieciocho caballos castrados de raza Criollo Argentino, clínicamente normales y con condiciones de alimentación y manejo similares. Los animales tenían una edad promedio de 12,5 ( $\pm 6,3)$ años de edad. Únicamente se incluyeron caballos con recuentos plaquetarios superiores a $100 \times 10^{3}$ plaquetas $/ \mu \mathrm{L}$. 


\section{ANTICOAGULANTES}

Se emplearon tubos con los anticoagulantes citrato de sodio (CS) (citrato de sodio 12,35 mg, ácido cítrico 2,21 mg (Equiv. 3,2\% CS) (BD Vacutainer ${ }^{\circledR}$ glass plasma tube, 13 x 75 mm x 4,5 mL. Lt. Blue. NJ, USA), y ácido citrato dextrosa (ACD), solución A (ACD-A) (citrato trisódico, 22,0 g/L, ácido cítrico, 8,0 g/L y dextrosa 24,5 g/L (BD Vacutainer $^{\circledR}$ glass whole blood tube, 16 x $100 \mathrm{~mm}$ x 8,5 mL. Yellow. NJ, USA).

\section{COLECCIÓN DE LAS MUESTRAS DE SANGRE}

Se extrajo sangre de la vena yugular mediante un catéter mariposa $21 \mathrm{G}$ (Terumo, Leuven, Bélgica), la que fue colectada en dos volúmenes independientes en tubos con anticoagulante en el siguiente orden: $112 \mathrm{~mL}$ en tubos de $4,5 \mathrm{~mL}$ con $\mathrm{CS} 3,2 \%(\mathrm{w} / \mathrm{v})$ y $140 \mathrm{~mL}$ en tubos de $8,5 \mathrm{~mL}$ con ACD-A, relación sangre final: ACD-A 10:2. La sangre anticoagulada fue transportada y mantenida en nevera con hielo durante una hora hasta su procesamiento. Esto con el propósito de controlar la activación espontánea de las plaquetas ex vivo e inferir las potenciales diferencias de los PRP al anticoagulante utilizado en la recolección de la sangre total. La sangre de un tubo elegido al azar con cada anticoagulante fue utilizada para hacer hemogramas basales.

\section{PREPARACIÓN DE LOS CONCENTRADOS DE PLAQUETAS}

La sangre de cada uno de los caballos fue procesada mediante el método de doble centrifugado en tubo descrito previamente (Argüelles y col 2006). Este protocolo de preparación manual de PRP-P ha sido evaluado previamente por otros investigadores (Vendruscolo y col 2012). Sangre total mezclada con cada uno de los anticoagulantes fue centrifugada a $120 \mathrm{~g}$ durante 5 minutos. El 50\% del volumen plasmático adyacente a la capa leucocitaria fue colectado en tubos de $10 \mathrm{~mL}$ siliconados sin aditivo. Estos tubos fueron centrifugados a $240 \mathrm{~g}$ durante 5 minutos. Luego se recogió el 25\% inferior (Argüelles y col 2006) de la fracción plasmática de la parte inferior de cada tubo. Esta fracción fue denominada PRP. La fracción superior restante (75\%) fue considerada PPP (figura 1). El PRP y PPP fueron evaluados inmediatamente.

\section{ANÁLISIS HEMATOLÓGICO}

Un hemograma automatizado (Celltac- $\alpha$ MEK 6450, Nihon Kodhen, Japón) fue realizado por duplicado a la sangre total, PRP y PPP, con cada anticoagulante. Se realizaron recuentos de plaquetas $\mu \mathrm{L}(\mathrm{PLT} / \mu \mathrm{L})$ y determinación de VPM (volumen plaquetario medio) y ADTP (amplitud de distribución del tamaño de las plaquetas) para estimar la activación plaquetaria. Estos parámetros plaquetarios se correlacionan con la activación de las plaquetas y

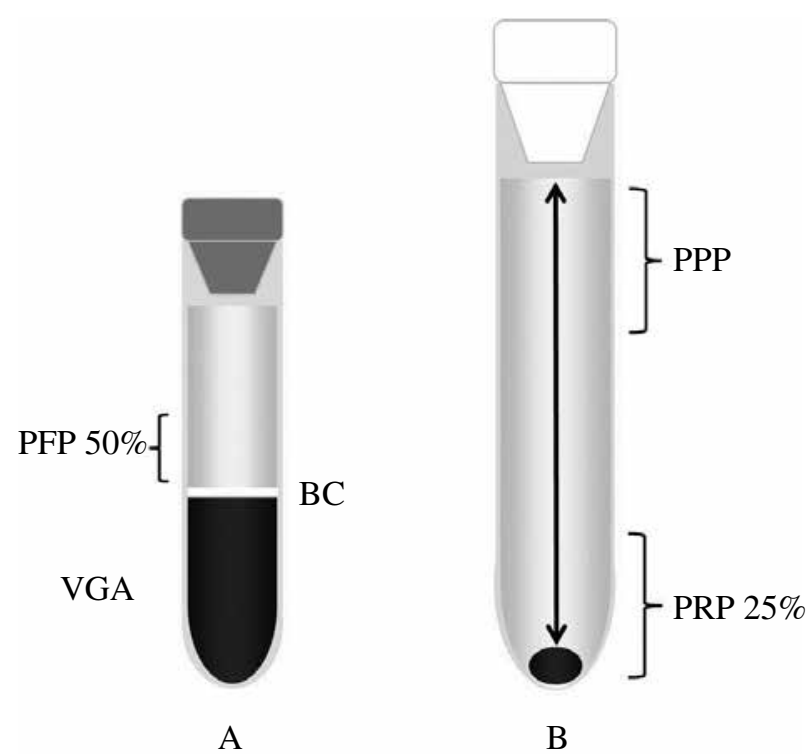

Figura 1. Representación esquemática de las fracciones de plasma obtenidas con el protocolo del método de tubo. El tubo de la izquierda (A) contiene primera fracción de plasma (50\%) (PFP) obtenida por el método de centrifugación simple en tubo. Tubo de la derecha (B) contiene plasma rico en plaquetas (PRP) obtenido por segunda centrifugación. BC: buffy coat. VGA: volumen globular aglomerado.

Schematic representation of the plasma fractions obtained with the tube method protocol. The tube on the left (A) contains the first fraction of plasma (50\%) (PFP) obtained by the single centrifugation tube method. The right tube (B) contains platelet rich plasma (PRP) obtained by second centrifugation. BC: buffy coat. VGA: packed cell volumen.

con la presencia de formas inmaduras y grandes en la circulación, respectivamente (Vasconcelos y col 2003). Se efectuó recuento de leucocitos/ $\mu \mathrm{L}$ y recuento absoluto (células/ $\mu \mathrm{L}$ ) y relativo $(\%)$ de linfocitos $(\mathrm{LI})$, monocitos (MO), eosinófilos (EOS), neutrófilos y basófilos (GRA). También se determinó la concentración de eritrocitos $/ \mu \mathrm{L}$.

\section{ANÁLISIS ESTADÍSTICO}

Los datos fueron analizados con el paquete estadístico SPSS 18.0 (IBM, Chicago, IL, USA). Las variables con distribución normal (Kolmogorov-Smirnov, P > 0,05) fueron evaluadas mediante pruebas paramétricas. Los datos sin distribución normal fueron transformados matemáticamente. Cuando no hubo distribución normal las variables fueron evaluadas con pruebas no paramétricas.

Las variables de respuesta fueron evaluadas para conocer la influencia de los anticoagulantes, CS y ACD-A. De manera general, cuando las variables normales fueron comparadas entre dos componentes sanguíneos se utilizó la prueba $t$-Student para datos independientes. Cuando se compararon más de dos medias se utilizó un ANOVA de una vía y una prueba Tukey post hoc. Las variables no paramétricas observadas entre dos grupos fueron analizadas con una prueba U de Mann-Whitney (UM-W). Cuando se 
evaluaron más de tres grupos con distribuciones no paramétricas se utilizó la prueba de Kruskal-Wallis seguida por pruebas post hoc de UM-W. Se realizaron correlaciones generales y específicas para las variables de cada componente hematológico mediante la prueba de correlación de rangos de Spearman $\left(r_{s}\right)$. Un valor de $\mathrm{P} \leq 0,05$ fue aceptado como significativo para todas las pruebas.

\section{EFICIENCIA DE LA COLECCIÓN DE PLAQUETAS EN PRP}

La eficiencia de colección de plaquetas se determinó mediante la fórmula de Weibrich y col (2003): (volumen de PRP x recuento de plaquetas en PRP/volumen de sangre total $\mathrm{x}$ recuento de plaquetas en sangre total) $\mathrm{x} 100$.

\section{RESULTADOS}

\section{RESULTADOS HEMATOLÓGICOS}

Los dos anticoagulantes produjeron PRP con leucocitos reducidos y recuentos de PLT significativamente mayores ( $\mathrm{P}<0,001)$, comparados con sangre total y PPP, en todos los casos. Los recuentos de PLT y leucocitos con CS y ACD-A en los componentes hematológicos homólogos fueron similares. Por un lado, las PLT fueron 2,7x en el PRP obtenido con CS, y 2,9x en PRP obtenido con ACD-A, comparadas con las PLT basales en sangre total. Además, los recuentos de leucocitos en PRP fueron ligeramente más altos que en sangre total, pero la concentración de estas células fue menor para el PRP obtenido con CS comparado con el mismo componente hematológico obtenido con ACD-A. Los leucocitos fueron 1,1x y 1,2x en PRP obtenido con CS y ACD-A, respectivamente, comparados con sangre total. No se observaron diferencias significativas para VPM y ADTP entre PRP y sangre total. Sin embargo, sí hubo diferencias (con significancias variables) entre los PRP y PPP en todos los casos. El factor de dilución de cada anticoagulante no influyó significativamente en los conteos celulares. Los recuentos celulares de los componentes sanguíneos homólogos obtenidos con CS y ACD-A se presentan en el cuadro 1.

\section{EFICIENCIA DE RECOLECCIÓN DE PLAQUETAS EN PRP}

La eficiencia de recolección de plaquetas en PRP obtenido con CS fue 19,7\% y en PRP obtenido con ACD-A fue $21,1 \%$. Sin embargo, no difirieron estadísticamente.

\section{CORRELACIONES}

Se observaron correlaciones significativas moderadas entre los conteos de PLT y leucocitos ( $\rho$ : 0,73, P < 0,01), conteos de leucocitos y LI $(\rho: 0,76, \mathrm{P}<0,01)$, conteos de PLT y LI ( $\rho: 0,60, P<0,01)$ y una correlación negativa moderada entre conteos de leucocitos y $\operatorname{ADTP}(\rho:-0,65$, $\mathrm{P}<0,01)$.

Cuadro 1. Resultados de las variables hematológicas para cada uno de los anticoagulantes estudiados y sus respectivos componentes sanguíneos. Los datos son presentados como medias ( \pm d.s.).

Results of the hematological variables for every anticoagulant studied and their respective blood components. Data are presented as means ( \pm s.d.).

\begin{tabular}{|c|c|c|c|c|c|c|}
\hline \multirow{3}{*}{ Variable } & \multicolumn{6}{|c|}{ Componente hematológico } \\
\hline & \multicolumn{2}{|c|}{ Sangre total } & \multicolumn{2}{|c|}{ Plasma pobre en plaquetas } & \multicolumn{2}{|c|}{ Plasma rico en plaquetas puro } \\
\hline & $\mathrm{CS}$ & ACD-A & $\mathrm{CS}$ & ACDA & $\mathrm{CS}$ & ACD-A \\
\hline PLT $\left(10^{3} / \mu \mathrm{L}\right)$ & $143,8(19,4)^{\mathrm{a}}$ & $137,0(21,3)^{\mathrm{a}}$ & $111,0(22,6)^{b}$ & $112.6(23.7)^{b}$ & $390,6(57,6)$ & $399,1(62,8)$ \\
\hline VPM (fL) & $3,8(0,4)$ & $3,8(0,4)$ & $3,6(0,3)$ & $3,7(0,4)$ & $4,1(0,6)^{\mathrm{c}}$ & $4,2(0,6)^{\mathrm{c}}$ \\
\hline $\operatorname{ADTP}(\%)$ & $16.5(0.5)^{\mathrm{c}}$ & $16,3(0,5)^{\mathrm{c}}$ & $17,8(0,5)$ & $17,6(0,7)$ & $16,8(0,5)^{\mathrm{c}}$ & $16,5(0,6)^{c}$ \\
\hline Leucocitos $\left(10^{3} / \mu \mathrm{L}\right)^{*}$ & $8,4(1,7)^{\mathrm{d}}$ & $7,9(2,0)^{\mathrm{d}}$ & $0,1(0,0)$ & $0,1(0,0)$ & $9,5(3,0)^{\mathrm{d}}$ & $9,8(5,0)^{\mathrm{d}}$ \\
\hline $\begin{array}{l}\mathrm{LI}\left(10^{3} / \mu \mathrm{L}\right) \\
\mathrm{LI} \%\end{array}$ & $\begin{array}{r}2,5(0,5)^{\mathrm{d}} \\
31,5(7,6)^{\mathrm{d}}\end{array}$ & $\begin{array}{r}2,3(0,5)^{\mathrm{d}} \\
30,8(7,2)^{\mathrm{d}}\end{array}$ & - & - & $\begin{array}{r}3,8(1,6) \\
35,7(11,7)\end{array}$ & $\begin{array}{r}4,3(2,5) \\
46,5(6,1)\end{array}$ \\
\hline $\begin{array}{l}\text { MO }\left(10^{3} / \mu \mathrm{L}\right)^{*} \\
\text { MO } \%\end{array}$ & $\begin{array}{l}0,1(0,0) \\
1,6(0,5)\end{array}$ & $\begin{array}{l}0,1(0,1) \\
1,9(0,6)\end{array}$ & $\begin{array}{l}- \\
-\end{array}$ & - & $\begin{array}{l}0,2(0,1) \\
2,3(0,7)\end{array}$ & $\begin{array}{l}0,2(0,1) \\
2,1(1,0)\end{array}$ \\
\hline $\begin{array}{l}\operatorname{EOS}\left(10^{3} / \mu \mathrm{L}\right) \\
\operatorname{EOS} \%\end{array}$ & $\begin{array}{l}0,2(0,1) \\
2,6(1,3)\end{array}$ & $\begin{array}{l}0,2(0,1) \\
3,2(2,1)\end{array}$ & $\begin{array}{l}- \\
-\end{array}$ & - & $\begin{array}{l}0,2(0,1) \\
1,8(1,4)\end{array}$ & $\begin{array}{l}0,2(0,1) \\
3,6(1,9)\end{array}$ \\
\hline $\begin{array}{l}\text { GRA }\left(10^{3} / \mu \mathrm{L}\right) \\
\text { GRA } \%\end{array}$ & $\begin{array}{r}5,4(1,4) \\
64,0(8,9)\end{array}$ & $\begin{array}{r}5,0(1,2) \\
63,9(8,7)\end{array}$ & $\begin{array}{l}- \\
-\end{array}$ & $\begin{array}{l}- \\
-\end{array}$ & $\begin{array}{r}6,7(3,3) \\
61,0(11,8)\end{array}$ & $\begin{array}{r}4,5(2,4) \\
49,4(5,1)\end{array}$ \\
\hline Eritrocitos $\left(10^{6} / \mu \mathrm{L}\right)^{*}$ & $6,9(0,8) \mathrm{a}$ & $6,4(0,7) \mathrm{a}$ & $0,0(0,0) b$ & $0,0(0,0) b$ & $0,2(0,1)$ & $0,2(0,1)$ \\
\hline
\end{tabular}

ACD-A, ácido citrato dextrosa (Solución A); CS, citrato de sodio; LI, linfocitos; MO, monocitos; EOS, eosinófilos; GRA, granulocitos (neutrófilos y basófilos); PLT, plaquetas; VPM, volumen plaquetario medio; ADTP, amplitud de distribución del tamaño de las plaquetas. ${ }^{\text {a-d }}$ Significativamente diferente con a: PRP y PPP $(\mathrm{P}<0,001)$. b: PRP $(\mathrm{P}<0,001)$. c: PPP $(\mathrm{P}<0,05)$. d: PPP $(\mathrm{P}<0,001)$. *Datos presentados como mediana (rangos intercuartílicos). ACD-A, acid citrate dextrose (Solution A); CS, sodium citrate; LI, lymphocytes; MO, monocytes; EOS, eosinophils; GRA, granulocytes (neutrophils and basophils); PLT, platelets; MPV, mean platelet volume; PDW, platelet distribution width. ${ }^{\text {a-d }}$ Significantly different with a: $P R P$ and $P P P(P<0.001)$. b: PRP $(P<0.001)$. c: PPP $(P<0.05)$. d: PPP $(\mathrm{P}<0.001)$ * Data are presented as medians (interquartile ranges). 


\section{DISCUSIÓN}

En el presente estudio fueron evaluados el CS y ACD-A, que son los anticoagulantes más comúnmente utilizados para la preparación de PRP equino con fines terapéuticos (Schnabel y col 2007, López y col 2010, Carmona y López 2011, Castelijns y col 2011). El efecto de ambos anticoagulantes fue estadísticamente similar en relación con la composición celular resultante en el PRP (cuadro 1). De acuerdo con estos hallazgos, los recuentos de PLT y leucocitos no tienen utilidad como indicadores para la elección del anticoagulante más idóneo al momento de preparar PRP en caballos.

Los recuentos de PLT (2,7-2,9x comparados con sangre total) y leucocitos (1,1-1,3x comparados con sangre total) observados en PRP obtenido con ambos anticoagulantes coincidieron con los rangos esperados para PRP con leucocitos reducidos, también conocido como PRP-P (PLT 1,3-4x y leucocitos 0,5-2x) (Dohan Ehrenfest y col 2012, Carmona y col 2013 ${ }^{\text {b }}$, comparados con sangre total. Además se observó estabilidad en los parámetros plaquetarios VPM y ADTP en sangre total y PRP, independientemente del anticoagulante utilizado, lo que sugiere estabilidad en tamaño plaquetario, baja activación espontánea e integridad durante la manipulación (Vasconcelos y col 2003).

Aunque desde el puro concepto de la hematología se ha comprobado que el ACD-A produce una mejor estabilidad de los parámetros de activación plaquetaria en el tiempo, en comparación con el CS (Macey y col 2002), desde el punto de vista de la medicina regenerativa y según los resultados del presente estudio, se podría pensar que el uso de uno u otro de los anticoagulantes evaluados no representa una ventaja clínica aparente. Esto porque el PRP se emplea como una terapia autóloga, esta normalmente debería ser preparada en el mismo lugar que se encuentre el paciente. Por lo demás, no se puede desconocer un estudio realizado en PRP humanos (Lei y col 2009) en el que se halló que el PRP obtenido con ACD-A producía una mayor liberación de TGF- $\beta_{1}$ y mayor proliferación in vitro de células estromales de médula ósea humana en comparación con CS. Sin embargo, es necesario realizar estudios adicionales para constatar si algo parecido podría ocurrir con el PRP equino.

Una ventaja técnica aparente al momento de preparar PRP en caballos mediante el método descrito en este estudio, podría ser la utilización de tubos con ACD-A, en lugar de tubos con CS. Esto porque los tubos de ACD-A tienen mayor capacidad (casi el doble) para la recolección de sangre en comparación con los de CS. Esto lógicamente reduce la cantidad de tiempo, material y riesgo de contaminación a la hora de preparar PRP con esta técnica (Álvarez y col 2010). Sin embargo, los autores prefieren emplear tubos de CS para preparar PRP con finalidades clínicas, porque aparentemente han obtenido mejores resultados (datos sin publicar). Sin embargo, se recomienda el uso de cualquier anticoagulante hasta que se hayan publicado resultados clínicos o estudios in vitro de equinos más convincentes.

Las correlaciones encontradas en el estudio para recuentos de plaquetas y leucocitos apoyan la presunción que hasta el momento no se ha podido desarrollar un método, técnica o aparato que pueda concentrar plaquetas sin concentrar cantidades apreciables de leucocitos (Carmona y col 2013 ${ }^{b}$ ). Se considera que el plasma rico en plaquetas y leucocitos (PRP-L) (McCarrel y Fortier 2009, Dohan Ehrenfest y col 2012, Carmona y col 2013 ${ }^{\mathrm{b}}$ ) es el que concentra plaquetas 5 veces o más y leucocitos 2 veces o más los recuentos basales de plaquetas y leucocitos en sangre total. En el caballo se ha reportado en la sangre circulante en condiciones de normalidad recuentos (promedio \pm DS) para plaquetas $167 \pm 26 \times 10^{3} / \mu \mathrm{L}$, y para leucocitos $8,2 \pm$ $1,6 \times 10^{3} / \mu \mathrm{L}$ (Giraldo y col 2013).

Sin embargo, con el método manual evaluado en este estudio y en otros (Giraldo y col 2013) normalmente se produce un PRP-P con un predominio de leucocitos mononucleares, particularmente LI. Algo muy diferente a lo que sucede cuando se produce PRP-L con métodos semiautomatizados, en los que se concentra una gran cantidad de neutrófilos (Sutter y col 2004, Carmona y col $2013^{\mathrm{b}}$ ). Actualmente se sabe que un exceso de este tipo de células podría ser nocivo para los tejidos tratados con esta clase de PRP (McCarrel y col 2012). También, un hallazgo importante de este estudio fue la correlación negativa moderada entre los conteos de leucocitos y ADTP ( $\rho$ : $-0,65)$, lo que sugiere que a medida que se activan las plaquetas durante el proceso de centrifugación se disminuye la concentración de leucocitos. Hasta el momento no se tiene una explicación biológica para este fenómeno, pero se podría considerar que, como sucede en PRP bovino, el parámetro ADTP podría ser más sensible para indicar el grado de activación plaquetaria que el VPM (López y col 2012).

En cuanto a la eficiencia de colección de PLT obtenidas en este estudio, se pudo constatar que ninguno de los anticoagulantes pudo influir en esta variable. Sin embargo, los resultados obtenidos fueron mayores (20\%) en comparación con los resultados obtenidos en caballos Andaluces (7\%), con sangre citratada procesada mediante el método del tubo (Argüelles y col 2006). Estas diferencias podrían obedecer, por ejemplo, al hecho de que se han descrito diferencias raciales al momento de preparar PRP mediante el método del tubo (Giraldo y col 2013).

Para concluir, este estudio presenta nueva información acerca del efecto de los anticoagulantes CS y ACD-A para la elaboración de PRP equino. Los resultados obtenidos en el estudio confirman la hipótesis inicial que tanto CS como ACD-A no influyen en la concentración de PLT o leucocitos, ni en el VPM o el ADTP en el PRP equino. Sin embargo, es necesario desarrollar investigaciones adicionales que permitan conocer el efecto de ambos anticoagulantes relativos a la liberación de factores de crecimiento o su efecto clínico o biológico en el caballo. 


\section{AGRADECIMIENTOS}

Esta investigación fue financiada por la Vicerrectoría de Investigaciones y Postgrados de la Universidad de Caldas, Colombia. Los autores agradecen a la Escuela de Carabineros "Alejandro Gutiérrez" de la Policía Nacional de Colombia, Manizales, Colombia.

\section{REFERENCIAS}

Álvarez ME, CE Giraldo, JU Carmona. 2010. Contaminación bacteriana en concentrados de plaquetas de caballos. Arch Med Vet 42, 49-56.

Álvarez ME, C López, CE Giraldo, I Samudio, JU Carmona. 2011. In vitro bactericidal activity of equine platelet concentrates, platelet poor plasma, and plasma against methicillin-resistant Staphylococcus aureus. Arch Med Vet 43, 155-161.

Araki J, M Jona, H Eto, N Aoi, H Kato, H Suga, K Doi, Y Yatomi, K Yoshimura. 2012. Optimized preparation method of plateletconcentrated plasma and noncoagulating platelet-derived factor concentrates: Maximization of platelet concentration and removal of fibrinogen. Tissue Eng Part C Methods 18, 176-185.

Argüelles D, JU Carmona, J Pastor, A Iborra, L Viñals, P Martínez, E Bach, M Prades. 2006. Evaluation of single and double centrifugation tube methods for concentrating equine platelets. Res Vet Sci 81, 237-245

Carmona JU, C López, M Prades. 2009 a . Use of autologous platelet concentrates obtained by the tube method as a treatment for arthropathies in horses. Arch Med Vet 41, 175-179.

Carmona JU, M Prades, D Arguelles. 2009 ${ }^{\mathrm{b}}$. Autologous platelet concentrates as a treatment for soft tissue musculoskeletal lesions in horses. Arch Med Vet 41, 77-82.

Carmona JU, C López. 2011. Autologous platelet concentrates as a treatment for shoulder injury in a horse. J Equine Vet Sci 31, 506-510.

Carmona JU, C López, C Giraldo. 2011. Uso de concentrados autólogos de plaquetas como terapia regenerativa de enfermedades crónicas del aparato musculoesquelético equino. Arch Med Vet 43, 1-10.

Carmona JU, C López, IJ Samudio. 2013ª . Autologous platelet concentrates as an adjunctive treatment for chronic laminitis in a mare with pituitary pars Intermedia dysfunction. J Equine Vet Sci 33, 191-195.

Carmona JU, C López, JA Sandoval. 2013 ${ }^{\text {b }}$. Review of the currently available systems to obtain platelet related products to treat equine musculoskeletal injuries. Rec Pat Reg Med 3, 148-159.

Carter CA, DG Jolly, CE Worden, DG Hendren, CJM Kane. 2003. Platelet-rich plasma gel promotes differentiation and regeneration during equine wound healing. Exp Mol Pathol 74, 244-255.

Castelijns G, A Crawford, J Schaffer, GA Ortolano, T Beauregard, RKW Smith. 2011. Evaluation of a filter-prepared platelet concentrate for the treatment of suspensory branch injuries in horses. Vet Comp Orthop Traumatol 24, 363-369.

Davies TA, DL Drotts, GJ Weil, ER Simons. 1989. Cytoplasmic $\mathrm{Ca}^{2+}$ is necessary for thrombin-induced platelet activation. J Biol Chem 264, 19600-19606.

Dohan Ehrenfest DM, T Bielecki, A Mishra, P Borzini, F Inchingolo, G Sammartino, L Rasmusson, P A. Evert. 2012. In search of a consensus terminology in the field of platelet concentrates for surgical use: platelet-rich plasma (PRP), platelet-rich fibrin (PRF), fibrin gel polymerization and leukocytes. Curr Pharm Biotechnol 13, 1131-1137.

Dunkel B, DM Bolt, RK Smith, FM Cunningham. 2012. Stimulusdependent release of tissue-regenerating factors by equine platelets. Equine Vet J 44, 346-354.

Flatow FA, EJ Freireich. 1966. The increased effectiveness of platelet concentrates prepared in acidified plasma. Blood 27, 449-459.

Giraldo CE, C López, ME Álvarez, IJ Samudio, M Prades, JU Carmona. 2013. Effects of the breed, sex and age on cellular content and growth factor release from equine pure-platelet rich plasma and pure-platelet rich gel. BMC Vet Res 9 .
Iacopetti I, A Perazzi, V Ferrari, R Busetto. 2012. Application of plateletrich gel to enhance wound healing in the horse: a case report. $J$. Equine Vet Sci 32, 123-128.

Kingston JK, WM Bayly, DC Sellon, KM Meyers, KJ Wardrop. 2001. Effects of sodium citrate, low molecular weight heparin, and prostaglandin E1 on aggregation, fibrinogen binding, and enumeration of equine platelets. Am J Vet Res 62, 547-554.

Lei H, L Gui, R Xiao. 2009. The effect of anticoagulants on the quality and biological efficacy of platelet-rich plasma. Clin Biochem 42, 1452-1460.

López C, JU Carmona. 2014. Platelet-rich plasma as an adjunctive therapy for the management of a severe chronic distal limb wound in a foal. J. Equine Vet Sci 34, 1128-1133.

López C, JU Carmona, I Samudio. 2010. Uso de concentrados autólogos de plaquetas como tratamiento de una fractura escapular y una lesión del plexo braquial producidas por un disparo en un caballo. Arch Med Vet 42, 209-214.

López C, C Giraldo, J Carmona. 2012. Evaluación de un método de doble centrifugación en tubo para concentrar plaquetas bovinas: estudio celular. Arch Med Vet 44, 109-115.

Macey M, U Azam, D McCarthy, L Webb, ES Chapman, D Okrongly, D Zelmanovic, A Newland. 2002. Evaluation of the anticoagulants EDTA and citrate, theophylline, adenosine, and dipyridamole (CTAD) for assessing platelet activation on the ADVIA 120 hematology system. Clin Chem 48, 891-899.

Maia L, MV de Souza, JI Ribeiro Júnior, AC de Oliveira, GES Alves, L dos Anjos Benjamin, YFRS Silva, BM Zandim, JdCL Moreira. 2009. Platelet-rich plasma in the treatment of induced tendinopathy in horses: histologic evaluation. J Equine Vet Sci 29, 618-626.

Marx RE. 2004. Platelet-rich plasma: evidence to support its use. J Oral Maxillofac Surg 62, 489-496.

McCarrel T, L Fortier. 2009. Temporal growth factor release from platelet-rich plasma, trehalose lyophilized platelets, and bone marrow aspirate and their effect on tendon and ligament gene expression. $J$ Orthop Res 27, 1033-1042.

McCarrel TM, T Minas, LA Fortier. 2012. Optimization of leukocyte concentration in platelet-rich plasma for the treatment of tendinopathy. J Bone Joint Surg - Series A 94, e143.141-e143.148.

Monteiro SO, OM Lepage, CL Theoret. 2009. Effects of platelet-rich plasma on the repair of wounds on the distal aspect of the forelimb in horses. Am J Vet Res 70, 277-282.

Schnabel LV, HO Mohammed, BJ Miller, WG McDermott, MS Jacobson, KS Santangelo, LA Fortier. 2007. Platelet rich plasma (PRP) enhances anabolic gene expression patterns in flexor digitorum superficialis tendons. J Orthop Res 25, 230-240.

Sutter WW, AJ Kaneps, AL Bertone. 2004. Comparison of hematologic values and transforming growth factor- $₫$ and isulinlike growth factor concentrations in platelets concentrates obtained by use of buffy coat and apheresis methods from equine blood. Am J Vet Res 65, 924-930.

Tablin F, NJ Walker, SE Hogle, SM Pratt, JW Norris. 2008. Assessment of platelet growth factors in supernatants from rehydrated freezedried equine platelets and their effects on fibroblasts in vitro. $\mathrm{Am}$ $J$ Vet Res 69, 1512-1519.

Textor JA, JW Norris, F Tablin. 2011. Effects of preparation method, shear force, and exposure to collagen on release of growth factors from equine platelet-rich plasma. Am J Vet Res 72, 271-278.

Textor JA, F Tablin. 2012. Activation of equine platelet-rich plasma: Comparison of methods and characterization of equine autologous thrombin. Vet Surg 41, 784-794.

Vasconcelos E, AC Figueiredo, J Seghatchian. 2003. Quality of platelet concentrates derived by platelet rich plasma, buffy coat and Apheresis. Transf Apher Sci 29, 13-16.

Vendruscolo CP, AdM Carvalho, LF Moraes, L Maia, DL Queiroz, MJ Watanabe, ALM Yamada, ALG Alves. 2012. Evaluating the effectiveness of different protocols for preparation of platelet rich plasma for use in equine medicine. Pesquisa Vet Bras 32, 106-110.

Zucker MB, RA Grant. 1978. Nonreversible loss of platelet aggregability induced by calcium deprivation. Blood 52, 505-514. 